\title{
Adaptive Approach to Acoustic Car Driving Detection in Mobile Devices
}

\author{
P. AKSAMIT* \\ Opole University of Technology Faculty of Electrical Engineering, Automatic Control and Informatics \\ Prószkowska 76, 45-758 Opole, Poland
}

\begin{abstract}
The context awareness of mobile devices is broadly researched area as it improves the functionality, usability, safety of usage and intelligence of the device as perceived by its user. The special type of context is driving a car. The awareness of a mobile device whose user drives a car may allow to disable some features like making and taking phone calls and enable other features like e.g. navigation thus improving the safety of the user. The paper presents the results of a research on acoustic detection of car driving based on over $60 \mathrm{~h}$ of collected data. The modification of traditional $k$-nearest neighbors classification algorithm is proposed that allows for learning and adaptation of classifier configuration. The proposed approach significantly improves both the sensitivity and specificity of the classifier comparing to the classifier based only on offline training data. The challenges in performing the acoustic wave analysis using highly heterogeneous devices like mobile phones are discussed.
\end{abstract}

DOI: 10.12693/APhysPolA.124.381

PACS: $43.60 . \mathrm{Vx}, 43.60 .-\mathrm{c}, 43.60 .+\mathrm{d}, 43.60 . \mathrm{Mn}$

\section{Introduction}

Analysis of acoustic signals is used in wide range of practical applications from medical imaging [1] to diagnostics of industrial apparatus [2-6]. The research presented in this paper concentrates on using acoustic signals analysis to detect car movement. This topic belongs to a broader field of context awareness of mobile devices [7-10]. The car driving was previously targeted by several studies. Miluzzo et al. developed a system called CenceMe, where sound was classified using simple linear classifier [11]. However, the achieved tradeoff between specificity $(96 \%)$ and sensitivity $(68 \%)$ was far from satisfying for practical applications. Additionally, the research does not address the problem of heterogeneity of mobile devices and conditions in which the users use their phones, which would probably reduce the performance of the classification even further.

In paper the modification of traditional $k$-nearest neighbor classification algorithm [12] is proposed that is able to adapt to the differences in mobile phones characteristics and users environment. The proposed solution increases the specificity of the classifier leading to reduced battery's energy consumption.

\section{Acoustic signal characteristics}

The signal was registered by mobile phones microphone, at a sampling frequency of $16 \mathrm{kHz}$. The surrounding sound was registered in $90 \mathrm{~s}$ intervals, for $5 \mathrm{~s}$ in each cycle.

\footnotetext{
*e-mail: p.aksamit@po.opole.pl
}

As presented in Fig. 1, the time-frequency characteristics of acoustic signal differs between different activities of mobile devices user. For example, the signal of driving a car contains a stationary component of significant magnitude in $0-200 \mathrm{~Hz}$ frequency range, which results from an engine sound registered by mobile phone microphone. Mobile phone lying in a pocket during walking periodically scratches the clothes, which results in periodical increases of signal power in broad spectrum of frequencies. When a mobile phone lies on a table, no characteristic signal is recorded, except the sounds of the surrounding environment.

The differences in signal characteristics for specific activities allow to determine the current activity of mobile devices by classifying the vector of characteristic features extracted from the signal.

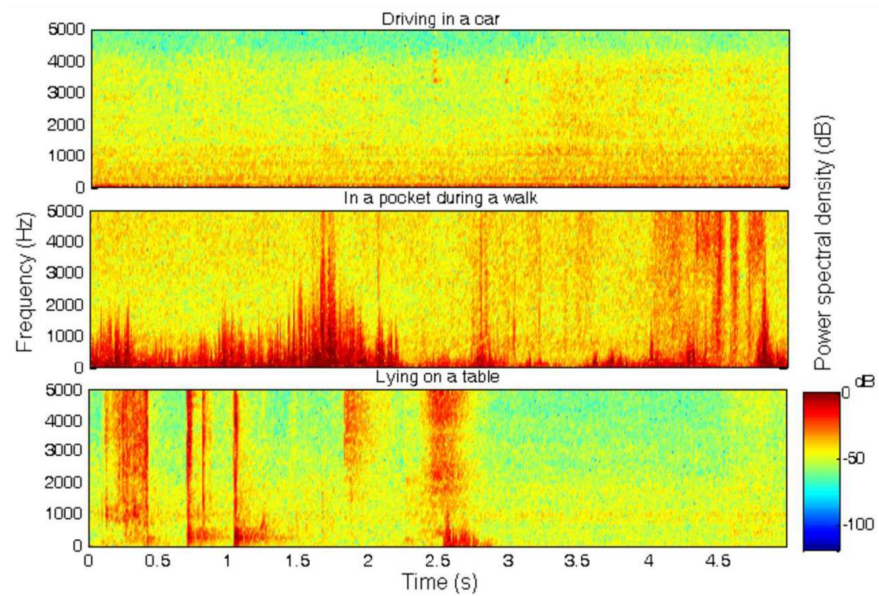

Fig. 1. Spectrograms of acoustic signals registered by mobile phone microphone in different situations. 


\section{Differences between devices}

Developing universal driving detection solution that would work on broad spectrum of mobile phones requires dealing with measurements made in highly heterogeneous, undefined and unpredictable conditions.

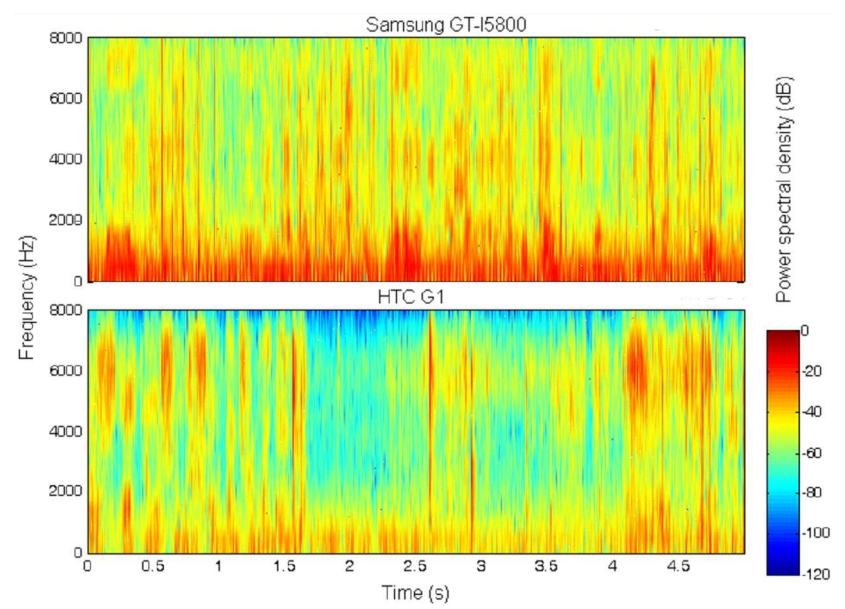

Fig. 2. Spectrograms registered at the same time, in the same conditions but on different devices.

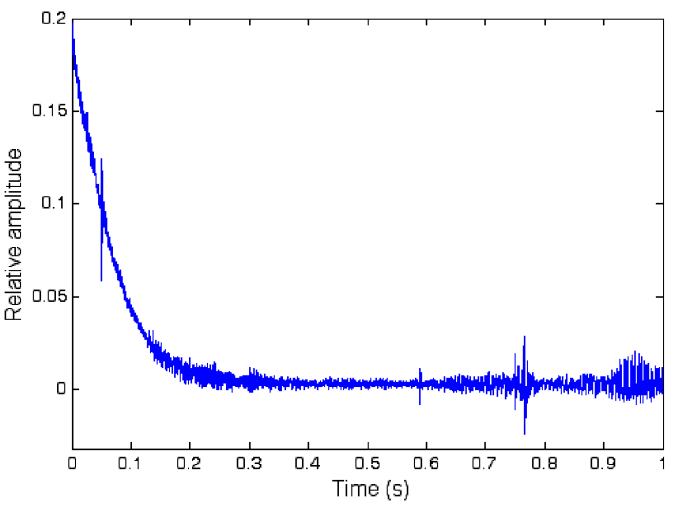

Fig. 3. Artifact after each start of signal registration on HUAWEI IDEOS U8500.

For example, Fig. 2 presents the spectrograms of two acoustic signals, registered at the same time, by two different mobile phones located at the same place in a driving car. The signal registered on the Samsung GT-I5800 has significantly higher power spectral density in the frequency range between $0 \mathrm{~Hz}$ and $2 \mathrm{kHz}$. In a large dataset of over $60 \mathrm{~h}$ of acoustic signals registered during the research, such differences occur frequently. They are an effect of many factors, such as different ways and places where microphones are mounted in different devices, different kinds of microphones used, or different characteristics of the filters.

Some mobile phones have also additional construction flaws that significantly influences the characteristics of registered acoustic signals. In Fig. 3, the signal registered on HUAWEI IDEOS U8500 (Android smartphone) is presented. After each activation of signal registration some capacity is discharging, which significantly influences the spectral characteristics of the signal sampled by $\mathrm{A} / \mathrm{D}$ converter.

\section{Classifier}

Even having large amounts of data, the heterogeneous nature of signals recorded by different devices makes it almost impossible to conduct single, offline training of a classifier, that would correctly work for all devices. Additionally, the mobile phone users drive hundreds different kinds of cars, which also differs in acoustic background during driving. Also when not driving, mobile phone users may spend their time in different environments, of different acoustic background - for example a car mechanic, that spends a lot of time close to working car engines.

This gives almost infinite number of possible combinations, which makes effective offline training virtually impossible. For those reasons, a modified version of well known $k$-nearest neighbors classification algorithm [12] was proposed, that would adapt its configuration to the conditions in which the mobile phones user uses his phone.

The vector of features extracted from the signal consisted of power spectral density up to $2 \mathrm{kHz}$, power of the signal and power in $0-2 \mathrm{kHz}$ to the power in $2-8 \mathrm{kHz}$ ratio.

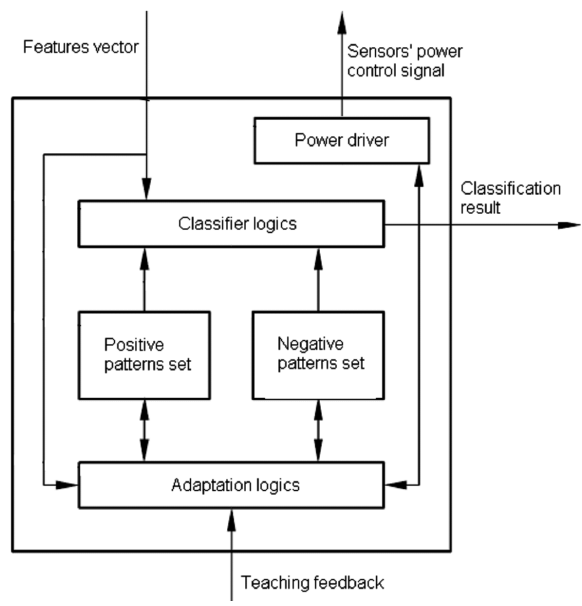

Fig. 4. Simplified schematic of proposed adaptive classifier.

The simplified schematic of proposed classifier is presented in Fig. 4. The core of the classifier are two sets of patterns, one representing driving (positive patterns set) and the other representing all other contexts in which the user uses his mobile phone (negative patterns set). When an input features vector is provided, the Euclidean distance is calculated between the given input vector and all patterns in both sets of patterns. Then, the distances are sorted in ascending order. If at least 2 of 3 smallest distances on the list are related to the patterns from 
positive patterns set, the classification result is positive, which means that the mobile phone user is driving a car.

After each evaluation of classification result, the adaptation logics takes the feedback signal confirming or negating the classifier outcome and modifies the patterns sets according to the scheme presented in Table.

Classifier adaptation scheme.

TABLE

\begin{tabular}{c|l|l}
\hline \hline $\begin{array}{c}\text { Feedback } \\
\text { positive }\end{array}$ & $\begin{array}{l}\text { Result positive } \\
\text { Add the classified } \\
\text { features vector to the } \\
\text { positive patterns set }\end{array}$ & $\begin{array}{l}\text { Result negative } \\
\text { 1. Remative patterns set } \\
\text { the pattern with the } \\
\text { shortest distance to } \\
\text { the classified features } \\
\text { vector } \\
\text { 2. Add the classified } \\
\text { features vector to the } \\
\text { positive patterns set }\end{array}$ \\
\hline $\begin{array}{c}\text { Feedback } \\
\text { negative }\end{array}$ & $\begin{array}{l}\text { 1. Remove from the } \\
\text { positive patterns set } \\
\text { the pattern with the } \\
\text { shortest distance to } \\
\text { the classified features } \\
\text { vector } \\
\text { 2. Add the classified } \\
\text { features vector to the } \\
\text { negative patterns set }\end{array}$ & $\begin{array}{l}\text { Add the classified } \\
\text { neatures vector to the }\end{array}$ \\
\end{tabular}

The teaching feedback signal may come from different sources, the simplest being the manual confirmation or negation of the classification result by the user. In the setup used for the experiment, the self-learning system was developed, which used accelerometer and GPS as a source of teaching feedback for sound classifier. Thus, the proposed solution does not require any engagement or attention from a user of a mobile phone.

\section{Results}

The effectiveness of the proposed approach was evaluated in an experiment, in which the mobile phone was located in an office near a fan of air conditioning device. In such conditions, the acoustic background registered by mobile phones microphone was very similar to acoustic background during driving a car. Classification result was evaluated repeatedly, in $90 \mathrm{~s}$ intervals.

The initial specificity of the offline trained classifier was $33 \%$. After one hour of operation with adaptation, the specificity approached $98 \%$, while without adaptation it remained at the level of $33 \%$. As the classifier is not a linear classifier, the rise of specificity in a given condition did not influence the sensitivity of the classifier, which remained at the same level of $97 \%$ before and after adaptation.

As each false positive result leads to activation of further sensors (in case of the experiment - accelerometer) to confirm or negate the obtained result, the low specificity of the classifier leads to increase in battery's energy usage. The proposed adaptive classifier reduced the energy usage from over $1.8 \% / \mathrm{h}$ to about $0.6 \% / \mathrm{h}$. As this translates to about 3 times longer operation of the device on fully charged battery, it is a significant improvement comparing to traditional offline trained classifier.

\section{Conclusion}

The paper addresses the problem of detecting users activity of driving a car by analyzing the sound registered by mobile phones microphone. In practical applications the challenge is to develop a solution that would work correctly on broad spectrum of different mobile phones as well as in different cars and other conditions in which users may use their phones.

The modified $k$-nearest neighbor classifier proposed in the paper adapts to the specific characteristics of users mobile phone, his car and other environments specific to the users daily activities. The result are higher specificity and sensitivity of classification that lead in effect to the significant reduction in battery's energy usage.

\section{References}

[1] C.C. Chen, J.S. DaPonte, M.D. Fox, IEEE Trans. Med. Imag. 8, 133 (1989).

[2] M. Lorenc, M. Szmechta, D. Zmarzły, T. Boczar, in: Proc. 2008 Int. Symp. on Electrical Insulating Materials (ISEIM), Yokkaichi, Mie, Eds. N. Hozumi, IEEJ, Tokyo 2008, p. 452.

[3] M. Szmechta, T. Boczar, P. Frącz, Acta Phys. Pol. A 120, 744 (2011).

[4] T. Boczar, D. Zmarzły, Official J. Am. Soc. Nondestr. Test. 62, 935 (2004).

[5] T. Boczar, D. Zmarzły, Insight - J. Brit. Inst. NonDestruct. Test. 45, 488 (2003).

[6] D. Wotzka, A. Cichoń, T. Boczar, Arch. Acoust. 37, 19 (2012).

[7] G.D. Abowd, A. Dey, R. Orr, J. Brotherton, Virt. Real. 3, 200 (1998).

[8] B. Schilit, N. Adams, R. Want, in: Proc. IEEE Workshop on Mobile Computing Systems and Applications (WMCSA 94), Eds. L.-F. Cabrera, M. Satyanarayanan, IEEE Press, 1994, p. 85.

[9] M. Philipose, K.P. Fishkin, M. Perkowitz, D.J. Patterson, D. Fox, H. Kautz, D. Hahnel, IEEE Pervasive Comput. 3, 50 (2004).

[10] D.J. Patterson, D. Fox, H. Kautz, M. Philipose, in: Proc. 9th IEEE Int. Symp. Wearable Computers, Ed. B. Werner, IEEE CS Press, 2005, p. 44.

[11] E. Miluzzo, N. Lane, K. Fodor, R. Peterson, H. Lu, M. Musolesi, S. Eisenman, X. Zheng, A. Campbell, in: Proc. 6th ACM Conf. on Embedded Network Sensor Systems, ACM, New York (NY), 2008, p. 337.

[12] K. Weinberger, J. Blitzer, L. Saul, J. Machine Learning Res. 10, 207 (2009). 